\section{RT-SHPM IMAGING OF PERMALLOY MICROSTRUCTURES AND GARNET FILMS USING NEW HIGH PERFORMANCE INSB SENSORS}

\section{A.Oral, M. Kaval, M. Dede and A. Sandhu}

Department of Physics, Bilkent University, 06533 Ankara, Turkey,

\author{
Tel: ++90312 290 1965, Fax: ++90312266 4579, e-mail: ahmet@fen.bilkent.edu.tr
}

${ }^{\dagger}$ Department of Electrical Engineering, Tokai University, 1117 Kitakaname

Hiratsuka 259-1292, Japan

Introduction

Scanning Hall Probe Microscopy (SHPM) [1] is a quantitative and non-invasive technique to image magnetic samples with high spatial and magnetic field resolution: $\sim 120 \mathrm{~nm} \&$ $6 \times 10^{-6} \mathrm{~T} / \mathrm{NHz}$ at room temperature. A sub-micron Hall probe is scanned over the sample surface to measure the surface magnetic fields using conventional scanning tunneling microscopypositioning techniques. We have developed new low noise, high performance InSb Hall sensor for Room Temperature Scanning Hall Probe Microscopy (RT-SHPM), which has a noise level of $6 \times 10^{-7} \mathrm{~T} / \mathrm{NHz}$.

$\underline{\text { Results }}$

The new sensors, which we have developed, show about $\times 10$ better noise performance compared to conventional GaAs, based sensors used in RT-SHPM. We used these new sensors to study magnetic domain structures of crystalline garnet films as shown in Figure 1 and $\mathrm{Ni}_{80} \mathrm{Fe}_{20}$ rectangular permalloy microstructures microfabricated by lift-off technique. The domain structures of the permalloy microstructures have been imaged by using SHPM, as the in-plane magnetic field was swept in various directions.

Conclusions

InSb Hall probes are shown to be highly sensitive and low noise alternatives to GaAs sensors for RT-SHPM. There seems to be more room for improving the spatial resolution and the noise of Hall probes to $<50 \mathrm{~nm}$ and to $1 \times 10^{-7} \mathrm{~T} / \mathrm{N} \mathrm{Hz}$ at room temperature.

[1] A. Oral et. al. Appl. Phys. Lett., 69, 1324 (1996),

A. Sandhu et. al. Jpn. J. Appl. Phys. 40(5B), L524 (2001)

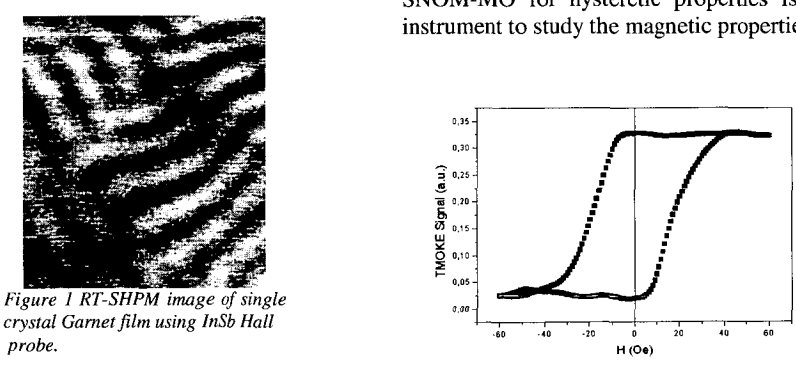

The magnetic material technical applications are progressively being miniaturized. As a consequence, the research community needs to develop new instrumentation to study the magnetic properties in a sub-micron or nanoscopic scale. In this paper we present the development of a Scanning Near-Field Optical Microscope (SNOM) devoted to the study of magnetic thin films. We have incorporated the capability of analyzing the light polarization to get magnetic information by means of the transverse magneto-optical Kerr effect (MO).

The SNOM-MO operates basically as an AFM, where the tip is made of a tapered optical fiber. The fiber is laterally Al coated, in order to have a sub-micron aperture at its end. The light source is a stabilized diode-laser. As the tip-sample distance is one order below the light wavelength, we are working in an evanescent wave mode. The sample's diffracted light is collected by an avalanche photodiode, giving us a near-field optical image, simultaneously to the topographic one. The lateral optical resolution is better than $100 \mathrm{~nm}$

By the light polarization analysis we can either measure the hysteresis loops with sub-micron spatial resolution or to construct the image point-by-point for some particular magnetic properties, as p.e., the coercivity or the differential susceptibility (see Fig. 1). Although the transverse Kerr effect is not quantitative for magnetic measurements, the sensitivity of the SNOM-MO for hysteretic properties is high and the SNOM-MO can become a powerful instrument to study the magnetic properties of thin films in a sub-micron spatial scale.

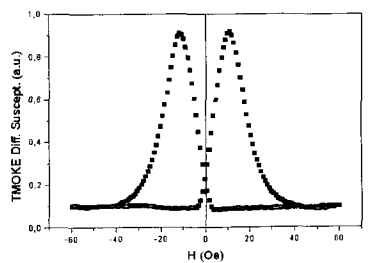

Figure 1) TMOKE Hysteresis (a) and Differential Susceptibility (b) loops measured by the SNOM-MO on an amorphous $\mathrm{CO}_{70.4} \mathrm{Fe}_{4.6} \mathrm{Si}_{15} \mathrm{~B}_{10}$ thin film, using a sub-micron aperture tip.

This work was supported by the Brazilian Agency FAPESP. 